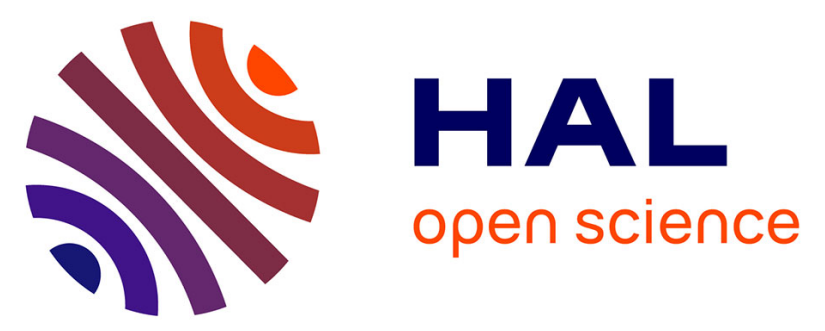

\title{
Optimisation of catalysts coupling in multi-catalytic hybrid materials: perspectives for the next revolution in catalysis
}

\author{
Egon Heuson, Renato Froidevaux, Ivaldo Itabaiana, Robert Wojcieszak, \\ Mickaël Capron, Franck Dumeignil
}

\section{To cite this version:}

Egon Heuson, Renato Froidevaux, Ivaldo Itabaiana, Robert Wojcieszak, Mickaël Capron, et al.. Optimisation of catalysts coupling in multi-catalytic hybrid materials: perspectives for the next revolution in catalysis. Green Chemistry, 2021, 23 (5), pp.1942 - 1954. 10.1039/d0gc04172f . hal-03219042

\section{HAL Id: hal-03219042 \\ https://hal.science/hal-03219042}

Submitted on 6 May 2021

HAL is a multi-disciplinary open access archive for the deposit and dissemination of scientific research documents, whether they are published or not. The documents may come from teaching and research institutions in France or abroad, or from public or private research centers.
L'archive ouverte pluridisciplinaire HAL, est destinée au dépôt et à la diffusion de documents scientifiques de niveau recherche, publiés ou non, émanant des établissements d'enseignement et de recherche français ou étrangers, des laboratoires publics ou privés. 


\section{TUTORIAL REVIEW}

Received 00th January 20xx, Accepted 00th January 20xx

DOI: $10.1039 / \times 0 \times x 00000 x$

\section{Optimisation of catalysts coupling in multi-catalytic hybrid materials: Perspectives for the next revolution in catalysis ${ }^{\dagger}$}

Egon Heuson, ${ }^{\mathrm{a}^{*}}$ Renato Froidevaux, ${ }^{\mathrm{a}}$ Ivaldo Itabaiana Jr, ${ }^{\mathrm{b}, \mathrm{c}}$ Robert Wojcieszak, ${ }^{\mathrm{b}}$ Mickaël Capron ${ }^{\mathrm{b}}$ and Franck Dumeignil ${ }^{b^{*}}$

The search for the optimal coupling of several types of catalysts has inspired the development of multi-catalytic hybrid materials (MCHMs) featuring chemical and biological catalysts co-immobilised on the same support. This complex interdisciplinary strategy, located at the crossroads of chemistry, biology, and materials science, calls for a wide range of skills and offers access to MCHMs with diverse catalytic properties and applications. In particular, numerous organic and inorganic supports, both rigid or flexible, have been used to develop remarkable hybrid catalysts that exhibit synergetic effects and achieve yields and enantiomeric excesses unattainable through the separate use of catalyst constituents even in one-pot/one step processes. As the spearhead of hybrid catalysis, MCHMs concentrate the very essence of this field, requiring seamless communication and collaboration between chemists, biologists, materials scientists, and modelling specialists. Future developments in this area are expected to revolutionise catalysis and make it an inter- or even transdisciplinary research area.

\footnotetext{
a. Univ. Lille, Université de Liège, UMRT 1158 BioEcoAgro - Biotransformation/

Enzymes et Biocatalyse, F-59000 Lille, France.

b. Univ. Lille, CNRS, Centrale Lille, Univ. Artois, UMR 8181 - UCCS - Unité de Catalyse

et Chimie du Solide, F-59000 Lille, France.

Departamento de Engenharia Bioquímica - Escola de Química, Universidade

Federal do Rio de Janeiro, Cidade Universitária, Rio de Janeiro, RJ 21941-909,

Brazil.

+ Corresponding authors E-Mail addresses: egon.heuson@univ-lille.fr;

franck.dumeignil@univ-lille.fr
}

This article is dedicated to P.H. Dixneuf for his outstanding contribution to organometallic chemistry and catalysis. 


\section{Introduction}

The development of more eco-friendly chemical processes is recognised as a priority task. Moreover, climate change and the scarcity of fossil resources require us to completely rethink our consumption habits and production models. In line with the formalisation of the widely adopted concept of green chemistry by Anastas and Warner, ${ }^{1}$ chemists are looking for more efficient (i.e., cheaper, less energy-consuming, and more environmentally friendly) alternatives to conventional synthetic methods. Catalytic processes can meet most of these requirements and are characterised by the use of at least one particular chemical species (catalyst) designed to promote one or more specific reaction steps, either chemical (chemocatalyst, homogeneous or heterogeneous) or biological (biocatalyst, e.g., enzymes and cells). Today, $90 \%$ of chemical processes use at least one catalyst, and $30-40 \%$ of the global economy directly or indirectly depends on catalyst usage. ${ }^{2}$ Chemical and biological catalysts are widely used in industry, enabling a better control of the selectivity and specificity of a wide variety of chemical reactions for the customised formation of various target compounds by affecting reaction pathways and, hence, activation energies, while most often favouring kinetics. Generally speaking, catalysts are spaces where physicochemical environments exhibit properties different from those of conventional reaction media, promoting or allowing specific reaction events (catalytic act) and even enabling the creation of new reaction intermediates that facilitate and/or direct reactions. The diversity of catalysts provides access to an extremely wide range of molecular mechanisms with varying degrees of complexity. Their understanding enables a better control of reactions and catalytic process optimisation. It also enables the reasoned coupling of catalysts according to the so-called multi-catalytic reactions that benefit from the novel properties of the thus assembled catalytic systems. Historically, catalysts have been used mostly in a unitary manner, with processes involving several catalysts most often forming parts of multi-stages strategies. However, the last two decades have seen a significant surge of interest in multi-catalytic reactions that combine two or more catalysts to benefit from collaborative effects. ${ }^{3}$ While catalysts of different natures are very rarely used together as we previously demonstrated, hybrid catalysis, referring to the combination of a chemical catalyst and a biocatalyst, is most likely the most promising type of multicatalytic reactions, offering unprecedented diversity and efficiency, as evidenced by the reports published over the last few years. ${ }^{4-11}$ Still, this approach remains most complex to implement, calling on expertise from different fields (chemistry, biology, materials science, etc.). The prospects of this strategy have inspired the development of new ways of circumventing the limitations and inhibitions that inevitably arise when these two types of catalysts are forced to work together. Hybrid catalysts can be subdivided into several categories depending on their constituents and the employed coupling strategy. However, it should be noted that not every combination of a chemical catalyst and a biocatalyst necessarily results in hybrid catalysis. Indeed, the major interest of the latter lies in the possibility of performing reactions that would otherwise be difficult to carry out, producing rare or non-existent compounds, or at least greatly improving the properties of an existing reaction (e.g., selectivity, yield, atom efficiency, and energy efficiency). Thus, combinations of catalysts leading to the development of new chemo-biosensors ${ }^{12}$ or allowing the regulation of enzymatic activity ${ }^{13,14}$ cannot be considered as hybrid catalysts. On the other hand, considering the combination of catalysts of different types for catalytic reactions, three main categories defining the basis of this new discipline can be identified, namely (i) dynamic kinetic resolution reactions (and their derivatives), (ii) regeneration of co-substrates, and (iii) new strategies aimed at the

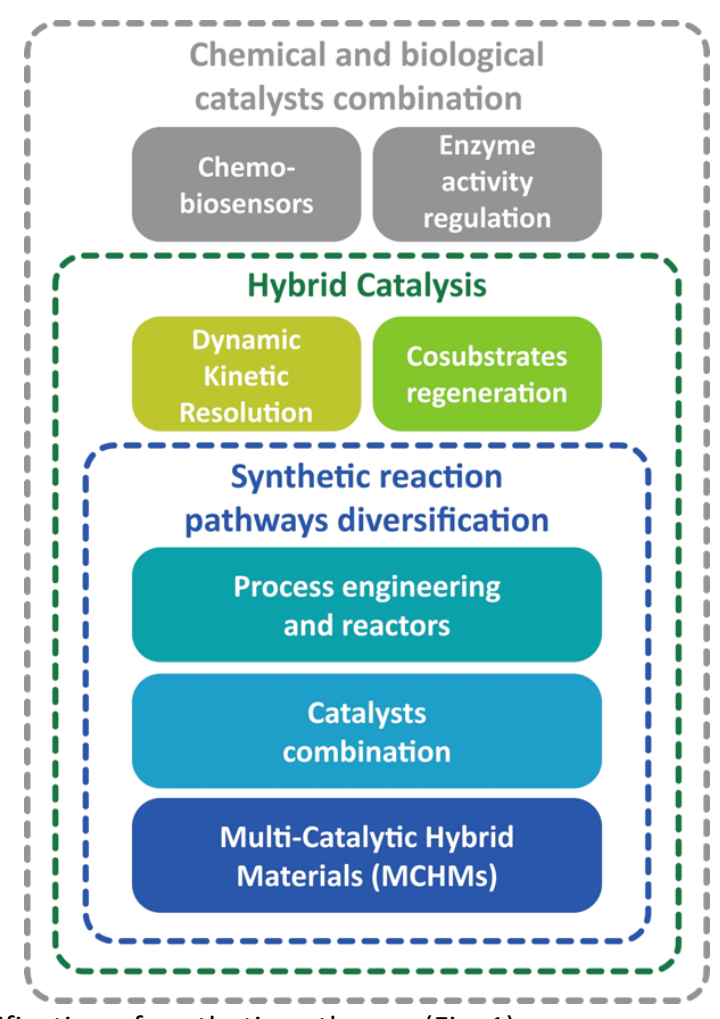

diversification of synthetic pathways (Fig. 1). 
Fig. 1. The different variants of hybrid catalysis and its positioning in the overal theme of combining chemo- and biocatalysts (figure translated from L'Actualité Chimique, $\mathrm{N}^{\circ} 454$, September 2020, with agreement).

It should be added that the last category can itself be subdivided into three families, each reflecting the strategy employed, i.e., (1) the development of new processes with several catalytic compartments, (2) the combination of several catalysts within the same reaction medium, and (3) the combination of catalytic centres within multi-catalytic materials. Irrespective of the strategy used, the goal of synthetic pathway diversification in hybrid catalysis remains the same, corresponding to the combination of several catalytic centres to allow the simultaneous (successive or parallel) transformation of compounds. To achieve this goal, several chemical functions can be modified at different points of the molecule to be transformed (reactant or substrate) during the reaction and be directly taken over by other catalysts to limit the number of purification steps, shift the overall reaction equilibrium in some cases, and ideally form difficult-to-synthesise compounds owing to the presence of new reaction intermediates. To obtain and modulate these properties, researchers employ various strategies allowing different catalyst combination types (Fig. 2). Historically, chemical and biological catalysts have been used in sequential processes and isolated in separate steps often separated by a purification step. These processes, which can be described as two-pots/two-steps (2P2S), ${ }^{3}$ are particularly costly in terms of atoms and energy and do not have the advantages offered by hybrid catalysis or even those of multi-catalytic reactions in general. Hybrid catalysis aims to integrate catalysts in one and the same reactor to maximise their interactions and uses other variants, starting with two-pot/one-step (2P1S) processes. As part of the process engineering strategy used to diversify synthetic routes, $2 \mathrm{P} 1 \mathrm{~S}$ processes employ catalysts that are separated only by a solid (porous) or liquid interface. The main advantage of this type of process is that it keeps the catalysts isolated from each other (which is particularly important if one catalyst directly poisons the other one) while allowing the diffusion of substrates from one reaction medium to another, which helps to avoid intermediate purification steps. It should also be noted that these processes allow the use of different reaction conditions for each catalyst, which considerably simplifies catalyst usage and reduces the need for common conditions. On the other hand, such catalytic systems are generally difficult to implement, require more complex/expensive equipment (membranes, multi-reactors, etc.), and above all, do not allow for synergetic effects between catalytic sites. Conversely, one-pot/two-step (1P2S) processes are more in line with a real catalyst association strategy. These processes involve the sequential or simultaneous introduction of two catalysts into the same reaction medium, with catalyst activities expressed successively by changing the reaction conditions ( $p H$, temperature, etc.) between each stage. The realisation of the overall process is thus greatly facilitated, but the interactions between the two catalysts are not optimal insofar as they do not perform their reactions concomitantly. This type of process is thus mainly chosen (1) when the substrate of one of the catalysts (or even the catalyst itself) exerts poisoning effects or (2) when common reaction conditions cannot be found. Examples include hybrid reactions involving a first step where an enzyme works at low temperature followed by a high-temperature step to enable the action of a chemical catalyst. Given that in this case, the two catalysts do not work together, the main limitation of this type of process is the total impossibility of establishing synergetic effects between the catalysts or even of shifting the reaction equilibrium using one of the two reactions, whereas 2P1S processes may allow such shifts and synergetic effects. To overcome these limitations and benefit from all advantages of hybrid catalysis, particularly for the conversion of complex substrate mixtures, researchers preferentially employ a true catalyst association strategy based on one-pot/one-step (1P1S) processes. These processes, which can be described as true hybrid catalysis, effectively combine several catalysts (working together throughout the reaction) within the same reactor. In this way, the different substrates and intermediates are continuously converted as soon as they appear, which allows one to shift equilibria and suppress inhibition to considerably increase the activities of the catalysts involved. Synergetic effects are sometimes observed between the different catalytic sites, in particular because of their proximity, facilitating material transfer and playing on the substrate gradients observed at the periphery of the catalysts. As a direct continuation of 1P1S process development, a third strategy is possible for the diversification of synthetic routes, further enhancing their benefits. The holy grail of hybrid catalysis, namely the physical combination of catalysts to afford a multicatalytic hybrid material (MCHM), relies on the very fine control of the arrangement of the catalytic sites in relation to each other to create additional effects between them (electrostatic, steric, etc.) and improve the diffusion of substrates within the material. 


\section{TUTORIAL REVIEW}

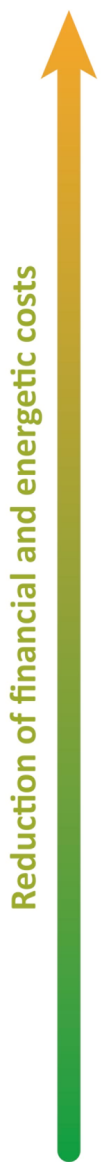

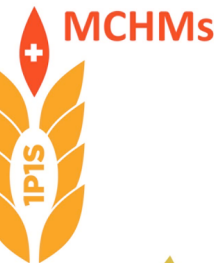

\section{Multi-Catalytic}

Hybrid Materials

One-Pot/One-Step

One-Pot/Two-Steps
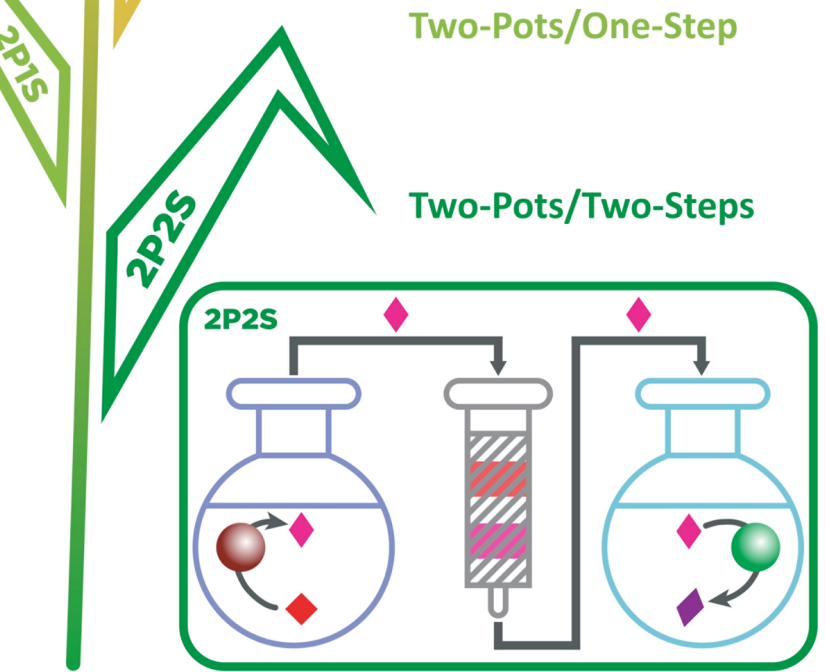
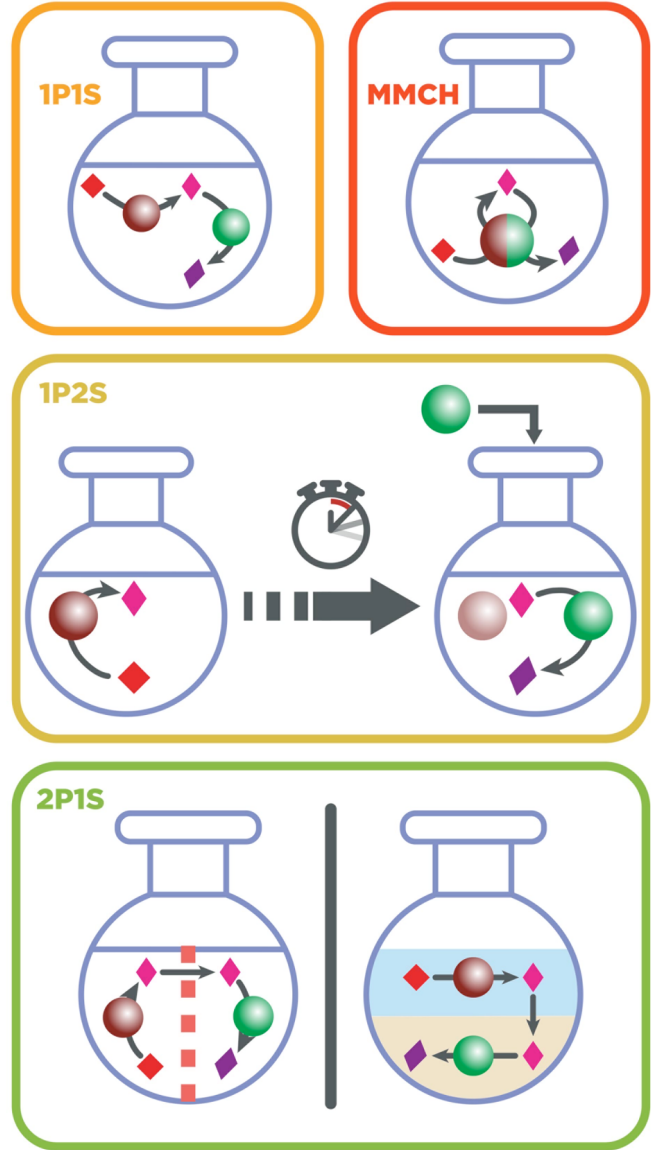

Fig. 2. Classification of processes combining chemical $\left({ }^{(}\right)$and biological $\left({ }^{\circ}\right)$ catalysts and used to set up hybrid catalytic reactions: two-pots/two-steps (2P2S), two-pots/one-step (2P1S), one-pot/two-steps (1P2S), one-pot/one-step (1P1S), and multi-catalytic hybrid materials (MCHMs). Substrate: ; intermediate: ; product: (Figure adapted from Heuson and Dumeignil, ${ }^{3}$ translated from L'Actualité Chimique, $N^{\circ} 454$, September 2020, with agreement).

\section{MCHMs}

The description of the first MCHM dates back to 2010, i.e., this disciplinary variant is one of the youngest in the world of catalysis. Here, we only discuss hybrid multi-catalyst materials and not those exclusively combining several chemical or biological catalysts, which have been developed over several decades. This late advent can be ascribed to two main reasons. First, the need for this type of catalytic entities was only felt once hybrid catalysis started to develop and demonstrate its advantages. However, this discipline dates back to the very early 2000s and saw its growth take off only in the 2010s. ${ }^{3}$ Second, even more than that of hybrid catalysis using separate catalyst coupling, the realisation of $\mathrm{MCHMs}$ requires a particularly diverse range of knowledge and skills. For example, one has to have a good knowledge and understanding of combined chemical and biological catalytic systems, in particular of their sensitivities and tolerances, as the proximity between these systems increases the likelihood of crosspoisoning and cross-inhibition. Moreover, it is essential to have a good knowledge of the material chosen for co-immobilisation, more particularly, of the interactions of this material with the catalytic systems involved, which further multiplies the number of constraints for the realisation of efficient MCHMs. Currently, only few teams bring together these different disciplines and, above all, make them interact, as interdisciplinarity is still, whatever is said and whatever efforts are made, a concept considered admirable but struggling to find its place in highly specialised research in a systematic manner. We will return to this point at the end of this article.

However, driven by the momentum of hybrid catalysis, the number of $\mathrm{MCHMs}$ has increased over the last decade, with 
about 20 examples described by December 2019. Before further describing these materials, their characteristics, as well as their advantages and limitations, we need to precise that we will focus here only on MCHMs combining a chemical catalyst and an enzyme. As MCHMs using whole cells belong more to the family of 2P1S processes, with their plasma membrane acting as a physical separator of the two reaction compartments, we deliberately do not cover these materials in the present article. However, such materials are equally efficient catalytic entities, as was shown, for example, by Turner et al., who combined a monoamine oxidase and $\mathrm{Pd}$ nanoparticles to effect the cyclic uprooting of 1-methyltetrahydroisoquinoline. ${ }^{15}$ In this work, the two catalysts were involved in two separate steps under very different operating conditions, and the host cell membrane responsible for the production of the enzyme was thus used as a physical separator. It should also be noted that when it comes to enzyme-based MCHMs, more than half of the described examples involve lipases. This predominance of lipases, which are quite different from other enzyme classes, can be explained by their specific properties such as tolerance to solvents and increased temperature, high stability, and, above all, the resulting ease of immobilisation on solid supports, all of which are assets for $\mathrm{MCHM}$ development. Herein, we try to present a maximum of examples for other enzyme classes to illustrate the diversity that MCHMs can offer. Finally, it should be added that almost all MCHMs reported so far employ monoatomic or nanoparticulate metal centres as chemical catalysts. Here, we consider only this type of catalysts for MCHM description.

\section{Unsupported catalyst combination}

\section{Protein shells}

When it comes to combining enzymes with metallic catalysts, several very different strategies can be used, starting with the seemingly simplest co-immobilisation of catalysts in the absence of a support. As enzymes are natural protein polymers, they can be directly used as supports for the immobilisation of metal particles in the same way as other polymer-type supports are used. Indeed, the numerous residues provided by enzymes offer many possibilities for interaction with metals. Moreover, this type of interaction is also found naturally in enzymes with a metal immobilisation function, for example, in those containing porphyrinic groups. Exploited at the biological level by metalloenzymes, these groupings offer a good source of inspiration as well as an increased variety of possibilities for researchers to incorporate metal centres into enzymes. However, the main difference between metalloenzymes and MCHMs is that the former need the metal to perform their catalytic activity, whereas MCHMs benefit from the coupling of several activities, each of which is linked to the presence of a different catalytic centre, either chemical or biological. In addition, the ease of production of enzymes as well as their biosourced origin make them excellent candidates for an industry eager to meet future environmental challenges.

Thus, when it comes to the immobilisation of metals within enzymes, the metal can be incorporated into a single protein (protein shell or PS) or be integrated into a mesh of several enzymes known as cross-linked enzyme aggregates (CLEAs).

The two strategies do not offer the same immobilisation possibilities and therefore result in different catalytic properties. In the case of PSs, the metal particles must be particularly small (a few nanometres at most) compared to their protein host. In this host (or in CLEAs), the metal centres are often randomly arranged within the polymer or on its surface, which makes particle size control less important (unless, of course, the catalytic activity sought for the particles thus dispersed depends on their size). Interestingly, PSs have long been used for the synthesis of size-controlled metal nanoparticles by a technique known as biomineralisation. ${ }^{16,17}$ This strategy has recently been used to realise the first example of such MCHMs based on the synthesis of Pt nanoparticles within the Streptococcus pneumonia aminopeptidase (PepA). ${ }^{18}$ This $\mathrm{Zn}$ aminopeptidase self-assembles into a well-defined tetrahedral dodecameric complex with external and internal diameters of $\sim 12$ and $6 \mathrm{~nm}$, respectively (Fig. 3), leaving sufficient room for nanoparticle growth. In addition, the PepA structure has channels of 1 and $3 \mathrm{~nm}$ diameter in the centre of the tetrahedral faces and on the edges, respectively, which allows the entry of the precursors required for the synthesis of Pt nanoparticles as well as the entry and exit of the substrates and products of the catalytic reactions.
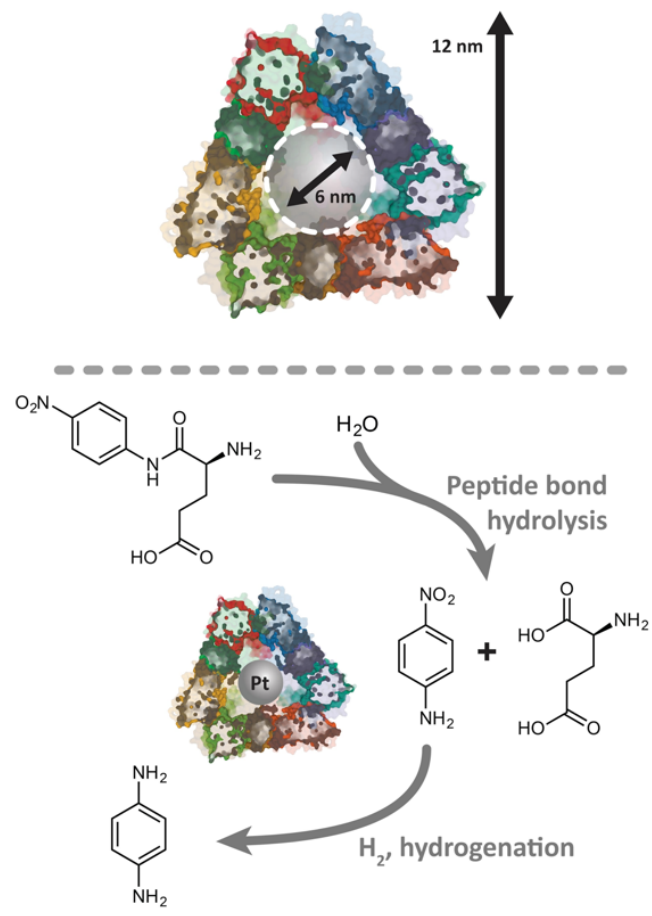

Fig. 3. The $6 \mathrm{~nm}$ cavity of the tetrahedral dodecamer of peptidase A (left) allows the controlled growth of $\mathrm{Pt}$ nanoparticles with a size of $0.9-3.2 \mathrm{~nm}$ depending on the incubation time of the protein envelope in the presence of a Pt" precursor $\left(\mathrm{K}_{2} \mathrm{PtCl}_{4}\right)$. The enzyme of this MCHM promotes the hydrolysis of peptide bonds, as illustrated here by the deprotection of $p$-nitroanilideglutamic acid at $37^{\circ} \mathrm{C}$ in the presence of Tris- $\mathrm{HCl}$ buffer $(\mathrm{pH} 7.5)$ and $\mathrm{ZnCl}_{2}$. Pt nanoparticles catalyse the parallel hydrogenation of chemical functions in the presence of a hydrogen source $\left(\mathrm{NaBH}_{4}\right.$ in this case), such as the reduction of the $p$-nitroaniline to $p$-phenylenediamine (right) (figure adapted from San et al., ${ }^{18}$ translated from L'Actualité Chimique, $\mathrm{N}^{\circ} 454$, September 2020, with agreement). 
This structure enables the controlled synthesis of very small nanoparticles with sizes of $0.9-3.2 \mathrm{~nm}$. The above example shows the importance of a very thorough knowledge of the structure of the enzyme used for the realisation of MCHMs, e.g., this enzyme has to be known in crystalline form and have a determined structure. At the end of the synthesis, the authors confirmed that nanoparticles were located inside the enzyme cavities and not on the enzyme surface. The thus modified enzymes had a lower-than-natural content of $\mathrm{Zn}$ ions, which indicated that these ions were partly exchanged for Pt ions within the active site. The resulting MCHM combined the catalytic activity of the enzyme with that of Pt nanoparticles and was used for the deprotection of $p$-nitroanilideglutamic acid followed by the reduction of the generated $p$-nitroaniline to $p$ phenylenediamine. The authors were thus able to study the individual activities of the two catalysts within the MCHM, showing that (according to the results of independent nanoparticle activity measurements) the activity of the enzyme and nanoparticles increased with decreasing nanoparticle size. The increased catalytic activity of Pt nanoparticles with decreasing size was ascribed to the concomitantly increasing exposure of active surface area (more Pt on the surface). In the case of the enzyme, the authors did not specify the reason for this increase, but it is logical to imagine that the decrease in nanoparticle size makes it possible to reduce interference with the enzyme active sites present within the cavity. Interestingly, the enzyme strongly stabilised the nanoparticles (i.e., extended their lifetime) relative to those modified by two classical chemical agents (citrate and Tween 80). This finding highlights the first synergistic effect that can appear within MCHMs, namely the stabilisation of one of the catalytic centres by the second one, which would not be possible in isolation. Moreover, the above MCHM also presented organic solvent-dependent activity, which implies that the diversity of catalytic conditions can widen the MCHM application scope and shows the importance of the combination of catalysts within the same material.

\section{Protein shell hybrids - organic polymer}

Although protein shells can be used to create embedded metallic nanoparticles, this approach only applies to the rare enzymes of sufficiently large size and with at least one type of cavity in which the nanoparticles can grow. As in the example presented above, one solution is to use enzymes that naturally assemble in the form of multimers with a given geometry to create the required cavity. However, such enzymes are also rare. This problem can be mitigated by complementing the enzyme structure with an organic polymer to create the required cavity, as exemplified by the synthesis of a lipasebased MCHM, in which case the authors were able to grow very small Pd nanoparticles in a well-controlled manner. ${ }^{19}$

\section{Surface immobilisation and CLEAs}

As a more common alternative, the growth of nanoparticles on the enzyme surface can be achieved by taking advantage of the roughness of the enzyme three-dimensional structure to guide the synthesis. This approach does not allow for structuring as precise as in the case of PSs, affording MCHMs in which the two catalysts are juxtaposed. The dimensions of nanoparticles can thus be more difficult to control for larger sizes, as shown by Filice et $a .^{20}$ In this study, the authors used Candida antarctica lipase B (CalB) as a carrier for the synthesis of Pd nanoparticles, simply adding it to a solution of Pd nanoparticle precursors in different organic solvents. Once the right precursor was selected (here, $\mathrm{Pd}(\mathrm{OAc})_{2}$ ) in the presence of different cosolvents, the authors observed enzyme precipitation and the formation of Pd nanoparticles on the enzyme surface. The thus produced nanoparticles had a bimodal size distribution, with the smallest ones having an average size of $1.3-3.5 \mathrm{~nm}$ (depending on the synthesis conditions), and the largest ones having a size of $4.5-6.8 \mathrm{~nm}$. The authors also tested precursors of various other metals and obtained $\mathrm{Ag}$ and $\mathrm{Au}$ nanoparticles of larger dimensions $(\sim 10 \mathrm{~nm})$ but with a monomodal size distribution. It should be noted that the organic solvent was reported to strongly influence the formation of nanoparticles and their size. This example explains why lipases are used in so many applications of this kind, as other enzymes are often totally denatured under such conditions. In fact, it is perhaps precisely the structural flexibility of lipases which, depending on the solvent, allows one to create asperities of varying amplitudes on their surface to influence nanoparticle size. Interestingly, the authors noted that under these conditions, the enzymes agglomerated to form a macro-polymer in which the nanoparticles were trapped. The formation of this organic network resulted in an MCHM that was particularly stable in aqueous solution, with no change in particle size and morphology observed over three months. This behaviour proved that the enzyme network acted not only as a physical carrier and a reducing agent during nanoparticle synthesis, but also as a stabilising agent. Despite agglomeration, up to $50 \%$ of lipase activity could be recovered, and the resulting MCHM was used in the synthesis of a nitroarene from an acetylated nitrophenol as well as for Suzuki-Miyaura and Heck coupling reactions.

Using the same model for the synthesis of MCHMs, one can further force enzyme cross-linking to increase the stability of the organic macro-polymer, e.g., via the formation of CLEAs, as mentioned previously. CLEAs are generated by the chemical coupling of enzymes to each other, very often using organic ligands such as glutaraldehyde to bind to specific residues of the enzyme. Although CLEAs are relatively easy to implement, they have major drawbacks such as difficult control of the polymerisation degree and the partial loss of enzymatic activity, as in the case of enzyme aggregation. It is indeed not uncommon that within the mesh, bonding between enzymes occurs on protein residues that are too close to the active site or are involved in protein folding, which precludes the establishment of an enzyme-substrate complex. In addition, there can be problems with the diffusion of substrates and products at the level of enzymes located in the centre of the mesh. Nevertheless, the high stability of CLEAs and their ease of synthesis make this enzyme immobilisation method very popular. CLEAs have been used for the synthesis of MCHMs, as described by Bäckvall et al.21,22 Finally, it is even possible to directly use metals for CLEA formation, e.g., Li et al. used copper 
to bind lipases together, taking advantage of the ability of peptide bonds to chelate copper via their nitrogen atom. ${ }^{23}$ The advantage of this method is that it can potentially be applied to any type of protein. The authors obtained a highly porous molecular mesh within which the enzymes were distributed but did not stop at CLEA formation, adopting a bottom-up - topdown approach featuring CLEA formation followed by fragmentation into unitary MCHMs composed of active copper nanoparticles onto which the enzymes were subsequently grafted (Fig. 4). During CLEA formation, the copper atoms were present as $\mathrm{Cu}^{2+}$ and had to be reduced to metallic $\mathrm{Cu}$ to induce catalytic activity. This fragmentation was enabled by the addition of polyvinyl pyrrolidone (a surfactant) to prevent the aggregation of metallic nanoparticles during reduction and form smaller isolated MCHMs. In this case, the enzymes were attached to the surface of nanoparticles, whereas conventionally, nanoparticles are attached to the enzyme surface. The authors demonstrated the presence of double catalytic activity by applying their MCHM to the conversion of $p$-nitrophenyl butyrate to $p$-aminophenol through hydrolysis followed by reduction, a model reaction used by all abovementioned lipase-containing examples. This type of MCHM is believed to rapidly find specific applications in the context of an industrial reaction to capitalise on these advances.

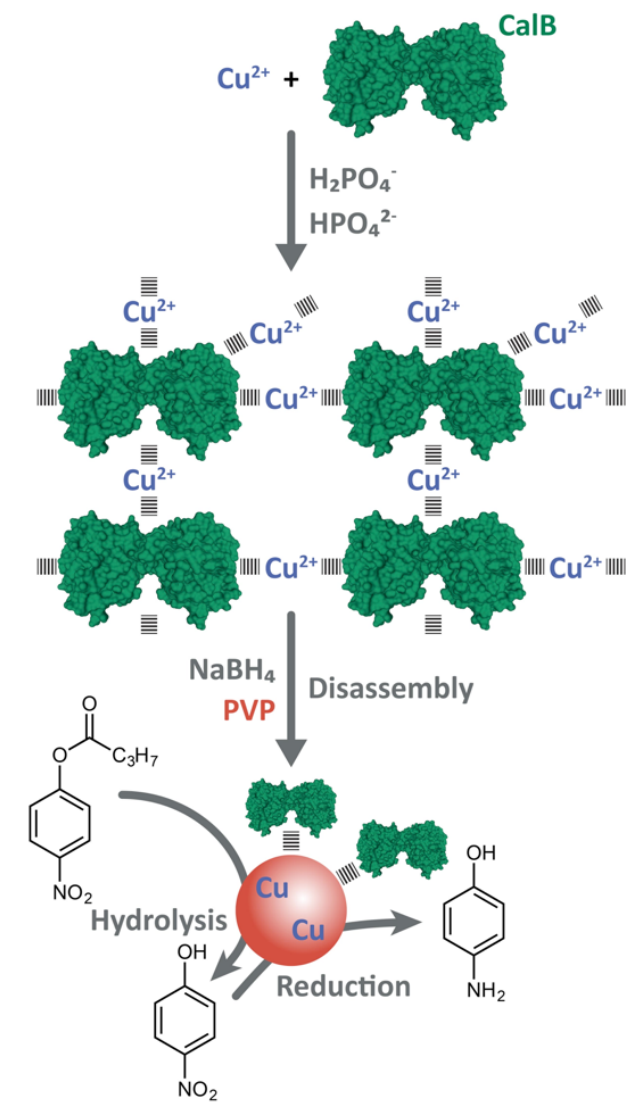

Fig. 4. Bottom-up/top-down approach utilising assembly/disassembly for the creation of an MCHM based on metallic $\mathrm{Cu}, \mathrm{CalB}$, and polyvinyl pyrrolidol. The $\mathrm{CalB}-\mathrm{Cu}^{2+}$ network was synthesised in the presence of phosphate ions, and disassembly was performed by the reduction of $\mathrm{Cu}^{2+}$ with $\mathrm{NaBH}_{4}$. The thus prepared $\mathrm{MCHM}$ was used for ester hydrolysis coupled with reduction (figure adapted from Li et al., ${ }^{23}$ translated from L'Actualité Chimique, $\mathrm{N}^{\circ} 454$, September 2020, with agreement).

\section{Use of inorganic carriers}

If the use of enzymes as matrices for the immobilisation of chemical catalysts has proven its efficiency through the examples presented above, the use of this strategy for MCHM construction remains underexplored. Inorganic supports are indeed much more represented, as exemplified by the use of those based on rigid materials for catalyst co-immobilisation, accounting for the vast majority of reported examples.

\section{Supports with a rigid structure}

The prominent representation of these media is probably due to their widespread usage for the separate immobilisation of enzymes and metals. In their co-immobilisation attempts, researchers have therefore logically turned to the above media in the first instance. Thus, many solid supports have been tested for the co-immobilisation of chemical and biological catalysts. ${ }^{4,8,24}$ Historically, zeolites were the first solid supports used for MCHM development. These crystalline inorganic materials have a microporous skeleton that makes them well suited for many applications, e.g., as dehydrating agents for the purification of various gases and the synthesis of heterogeneous catalysts for various reactions at the industrial level (epoxidations, isomerisations, etc.). ${ }^{25,26}$ The first MCHM reported in 2010 involves the coupling of glucose oxidase with a titanium silicate-1 (TS-1) zeolite. ${ }^{27}$ Currently, titanium-based zeolites are widely used for the oxidation of oxygenated functions such as alcohols and epoxides. The use of TS- 1 as an enzyme support therefore allows one to combine the above oxidation potential with enzymatic activity. In their study, the authors began by using glucose oxidase and TS- 1 zeolite in a 1P1S process, but with the two catalysts separated in solution. Although coupling was shown to be relatively functional for epoxide formation, the enzyme suffered (i.e., was eventually denatured) from contact with the produced $\mathrm{H}_{2} \mathrm{O}_{2}$ (dispersed in the medium) until its reduction by the metal catalyst. To mitigate this problem, the authors sought to reduce the path of $\mathrm{H}_{2} \mathrm{O}_{2}$ diffusion between the enzyme and the metal. To do so, they immobilised the enzyme on the zeolite surface and thus realised an MCHM for the efficient oxidation of different shortchain alcohols such as 2-butanol or 2-propanol, which was not possible with the free enzyme. This finding illustrates that when two catalysts are co-immobilised, a new synergistic effect can occur to extend the reaction scope beyond that attainable when the catalysts are used in tandem but in isolation. It should be noted that the strategy of enzyme immobilisation on the material surface is the one most widely selected for MCHM development because of its easier implementation compared to that of other more complex techniques. However, as specified by the authors, this strategy only partially limits inhibition by the substrate, with the enzyme still remaining in contact with the reaction medium, and the concentrations of products they could obtain remained anecdotal even in the case of the use of their new MCHM. A new innovative approach has been developed by Smeets et al., ${ }^{28}$ who immobilised glucose oxidase by encapsulation within the inorganic micelles of a TS-1 zeolite rather than by creating covalent bonds on the zeolite surface. In this approach, mesoporous spheres were formed by 
using an atomiser to pass the zeolite precursor solution as an aerosol. Once sprayed, the microspheres formed naturally and solidified upon drying. The enzyme introduced by simple impregnation was cross-linked to form a CLEA within the micelle, which prevented enzyme re-diffusion to the outside (Fig. 5).

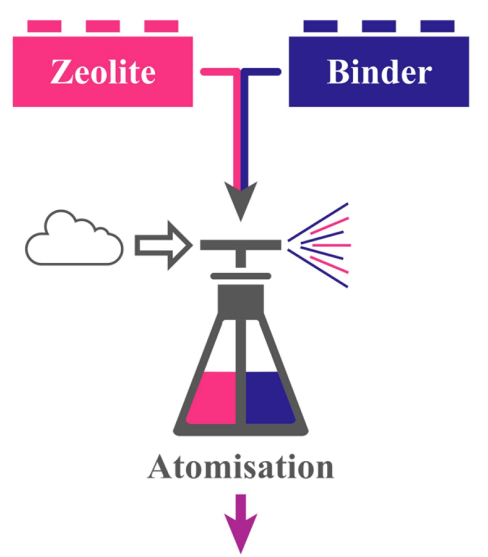

TS-1 zeolite

mesoporous spheres
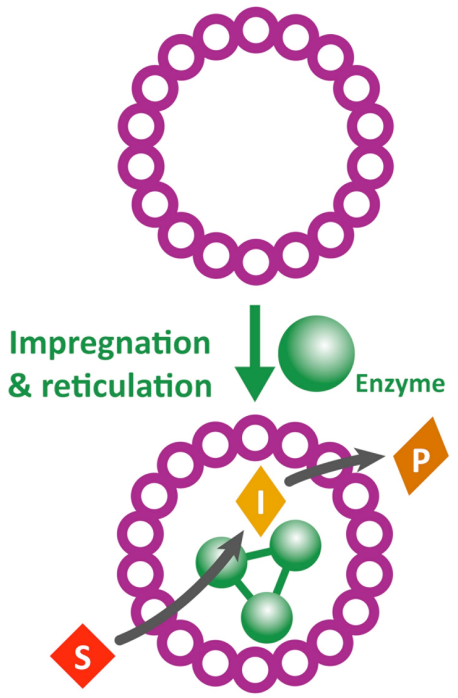

Multi-Catalytic

Hybrid Material conversion of slightly above $30 \%$ after $25 \mathrm{~h}$. In this case, the yield obtained using the MCHM was not much higher than that obtained with the two catalysts in free form, although the selectivity for glycidol increased ( $76 \%$ vs. $67 \%$ for separate catalysts). This limitation was also attributed to CLEA formation, which, as mentioned above, can induce significant enzyme denaturation, with only $50 \%$ of specific activity retained in this case. Nevertheless, this work highlights the potential of MCHMs for the realisation of more selective reactions through better control of product formation. It is also essential to quantify the electronic effects at the origin of the interactions appearing in these MCHMs and governing the new catalytic properties and selectivities if these interactions are to be exploited in the best possible way through the construction of MCHMs in a more reasoned manner.

In addition to zeolites, other silica-based materials such as mesoporous silica particles have also been used as supports for the creation of MCHMs. In most cases, enzymes were grafted onto the material surface. As a representative member of this MCHM family, we can mention the material recently synthesised by De Souza et al. via the covalent immobilisation of CalB on functionalised silica with supported $\mathrm{Pd}$ nanoparticles. ${ }^{29}$ This multi-catalytic material facilitated the dynamic kinetic resolution of $\alpha$-methylbenzylamine, which usually involves the use of several catalysts in separate steps or at higher concentrations. At a Pd loading of $1 \mathrm{wt} . \%$, the final enantiomeric excess exceeded $99 \%$, and conversion equalled $82 \%$, which resulted in increased productivity (2.21 vs. 0.76 mg.h ${ }^{-1} \cdot \mathrm{mg}_{\text {carrier }^{-1}}$ for Novozyme $435^{\circledR}$, a common commercial lipase). In addition, this new hybrid catalyst had a protein loading 15 times lower and an activity higher than those of currently employed commercial solutions, thus holding great promise for industrial applications. It should be noted that the same process was applied to Pd nanoparticles immobilised on a separate support, and the observed enantiomeric excess was lower than in the case above. However, unlike zeolites, as illustrated in the previous example, the active species are not constitutive of the support. This represents a certain advantage in terms of the spectrum of metals that can be used, but also causes release problems when the metal particles are not strongly bound to the material. A possible solution to this problem, suggested by Bäckvall et al., relies on the covalent coimmobilisation of the two catalysts in the core of material pores. ${ }^{30}$ This approach has led to the development of a particularly efficient MCHM that combines lipase with Pd nanoparticles and achieves conversions of up to $99 \%$ and an ee of $>99 \%$ for 1-phenylethylamine uprooting. However, this approach does not resolve the second limitation of this type of grafting onto an inert support, namely the effective control of the positioning of enzymes with respect to metal centres to limit their contact in the event that the enzyme inhibits the activity of the metal or vice versa. To circumvent this limitation, researchers tried to immobilise the metallic nanoparticles in the material pores while keeping the enzymes confined to the material surface. This goal can be achieved by modulating the hydrophobicity of the silica beads' surface using organic hydrophobic groups so that the enzyme is no longer covalently 
bound to the support but is rather held in place by hydrophobic interactions. ${ }^{31}$

Another strategy relies on the construction of a silica ball around a metal centre located at its core. ${ }^{32}$ By preserving the formation of pores sufficiently large for the diffusion of substrates and products towards the metallic nanoparticle, the authors constructed an efficient MCHM combining $\beta$ glucosidase with $\mathrm{Au}$ nanoparticles, although these nanoparticles are often inhibited by protein residues. Other non-silica inorganic supports (such as nanoflowers) were also tested for their ability to co-immobilise chemical catalysts and enzymes. These emerging materials appear to hold great promise for MCHM development, as they are increasingly used for the separate immobilisation of enzymes or metal catalysts, although only one example of coupling for medical and military diagnostic purposes has been reported so far. ${ }^{33}$

Finally, the use of carbonaceous organic supports should also be mentioned. First of all, we note the use of graphene sheets for the development of the first medically applicable MCHM, which was synthesised by exploiting the $\pi-\pi$ interactions offered by graphene to immobilise a haeme and glucose oxidase by covalent bonding. ${ }^{34}$ Benefiting from these interactions with the support, the haeme could oxidise arginine to nitrogen monoxide (NO), while the enzyme used glucose for the generation of $\mathrm{H}_{2} \mathrm{O}_{2}$ necessary for this oxidation. NO is now recognised as an anti-thrombotic molecule, as it is continuously generated by the endothelial cells lining the inner walls of all blood vessels. The presence of glucose and arginine readily available in blood makes this MCHM particularly useful for complementing this natural biosynthesis. The authors even showed that their MCHM could be integrated into a polyurethane matrix without any loss of catalytic properties, which facilitated integration into medical devices. The above study reveals how organic carriers, particularly carbonaceous sheets with high biocompatibility, can be used to realise efficient MCHMs for biological and medical applications. Carbon nitride $\left(\mathrm{C}_{3} \mathrm{~N}_{4}\right)$ foils have been recently used for the coimmobilisation of $\mathrm{Pd}$ nanoparticles and CalB. ${ }^{35}$ large amount of nitrogen atoms and the high proportion of weakly coordinated metal atoms in these composites, exceptional activity was obtained. At the same time, the noble metal loading could be minimised to reduce the cost and increase the environmental friendliness of these catalysts. Indeed, two-dimensional conjugate sheets comprising tris-s-triazine repeating units greatly facilitate the binding of metal (e.g., Pd, Pt, $\mathrm{Ag}, \mathrm{Au}$, or $\mathrm{Cu}$ ) particles in the matrix, which was used to directly incorporate nanoparticles into the material. Concerning the enzyme, rather than relying on covalent binding, the authors chose to take advantage of weak enzyme-material interactions to immobilise the enzyme in the form of sheets formed using glutaraldehyde in the same way as for the realisation of CLEAs. Interestingly, the authors observed that the activity of the enzyme for their model reaction (transesterification of ethyl hexanoate with benzyl alcohol) greatly increased. This result suggests that the physical and chemical interfaces of $\mathrm{C}_{3} \mathrm{~N}_{4}$ may affect the orientation and conformation of the immobilised CalB, contributing to the increased activity. In addition, free CalB easily aggregates in organic solvents, while the amphiphilic nature of $\mathrm{C}_{3} \mathrm{~N}_{4}$ allows for better dispersion and thus facilitates substrate-enzyme contact. $\mathrm{Pd}$ nanoparticles also showed significant activity for the reduction of benzaldehyde to benzyl alcohol, and the authors successfully used their MCHM for the production of benzyl hexanoate from benzaldehyde. Again, important limitations related to the protection of catalysts and their stabilisation over time were observed, leading to catalyst denaturation after several catalytic cycles, particularly at the enzyme level. As we have seen, the use of fixed-structure materials as MCHM supports only rarely allows one to protect the catalysts from each other or from the reaction medium, in particular because of the need to fix them to the surface. This disadvantage is compounded by the lack of selectivity and specificity associated with this type of structure, as discussed in the second part of this review, which are essential factors for catalytic performance regulation. ${ }^{36}$ order to circumvent these limitations, researchers have attempted to develop more flexible and even breathable materials.

\section{Use of flexible supports}

One of the most interesting properties of flexible nanostructures is the tunability of their size, shape, structure, chemical functions, and, hence, of the dispersion of catalysts within them. Metal-organic frameworks (MOFs) or their covalent counterparts, covalent organic frameworks (COFs), are the spearheads of this class of materials and have been successfully used to design new MCHMs. MOFs, otherwise known as porous coordination polymers, are synthesised by the self-assembly of metal ions or polyoxo-clusters ( $3 d$ transition metals, $3 p$ metals or lanthanides) with organic di- or polytopic ligands (carboxylates, nitrogen donors, sulfonates, or phosphonates) in the form of highly porous crystals (channels). ${ }^{37}$ The main advantage of MOFs for hybrid catalysis lies in their ability to form structures with almost unlimited variations in pore size, shape, and function. It should also be noted that some MOFs were reported to exhibit a certain breathability due to structural flexibility, thus meeting the performance criterion for hybrid catalysis. This particularity allows these to structurally adapt for the incorporation of elements of different dimensions. Of course, not all MOFs demonstrate this capacity to change their structural conformation, and only those that present this crucial ability make it possible to offer new properties with respect to the carriers listed in the previous chapter. The others, such as the UiO family for example, are thus more similar to rigid supports and are in fact just as interesting as the latter for the synthesis of MCHMs. Whatever their flexibility, such structural versatility is linked to the wide variety of metals and ligands that can be used for MOF synthesis as well as to the fact that MOFs can be post-functionalised at the level of metal centres and ligands ${ }^{38}$ by replacement of the latter after the synthesis stage. Several studies also describe the possibility of synthesising multi-metal and multi-ligand MOFs, even if these syntheses are difficult in practice. ${ }^{39}$ It is this structural and synthetic versatility that is particularly interesting for the development of multi-catalytic materials (Fig. 6). 


\section{TUTORIAL REVIEW}

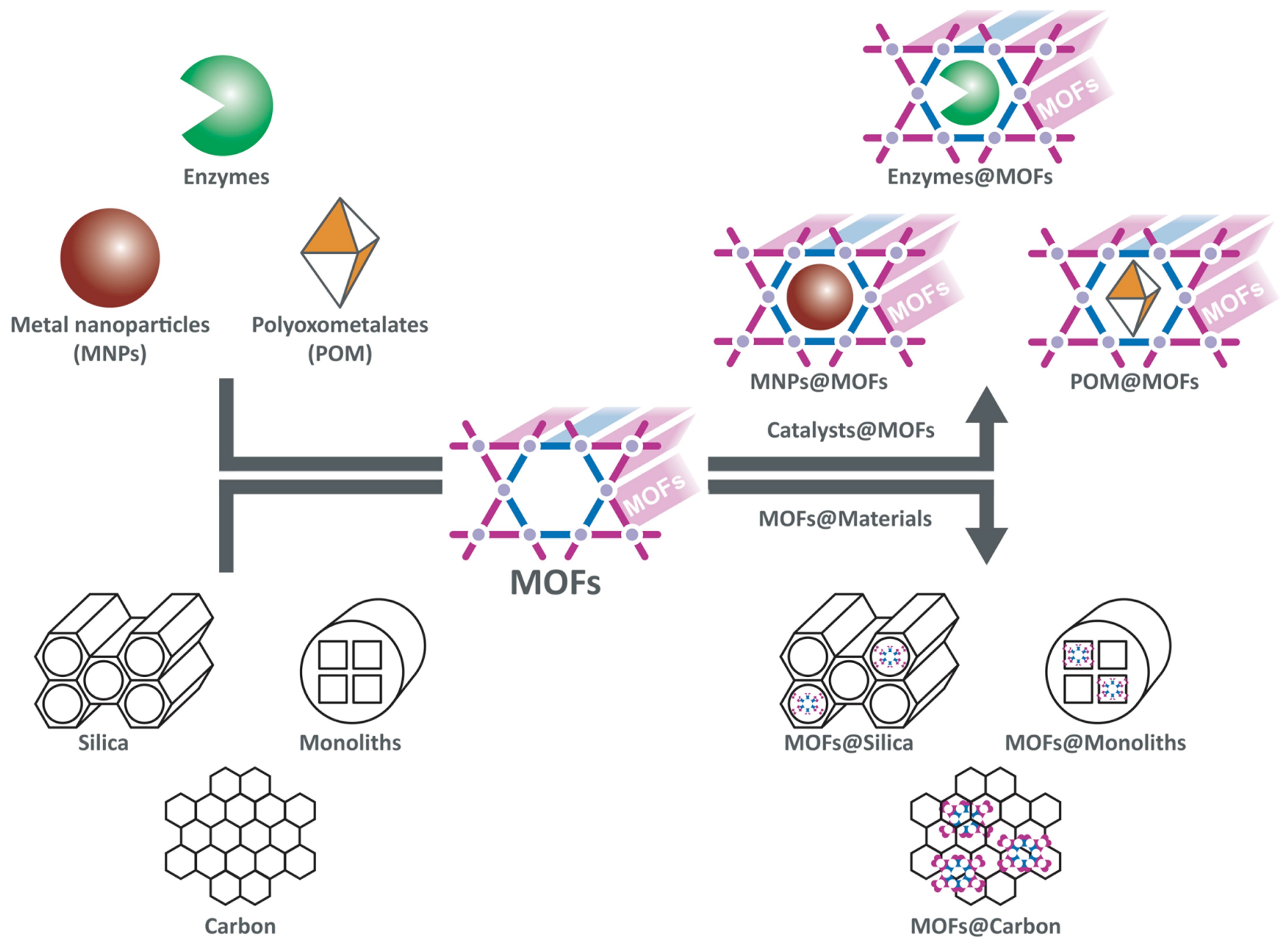

Fig. 6. Possibilities of combining MOFs with heterogeneous catalysts and supports to obtain new functional catalysts (figure adapted from Chen and Xu, ${ }^{40}$ translated from L'Actualité Chimique, $N^{\circ} 454$, September 2020, with agreement).

The first MOF-based MCHM was reported in $2017 .{ }^{41}$ In this example, nanoparticles were not used to carry out an additional synthesis step, but were rather employed for their peroxidaselike activity to convert the enzymatically produced $\mathrm{H}_{2} \mathrm{O}_{2}$ into $\mathrm{H}_{2} \mathrm{O}$. Kinetic studies of the reaction catalysed by nanoparticles immobilised within the MOF revealed a Mickaelian behaviour similar to that of an enzymatic peroxidase. Therefore, the authors measured the kinetic parameters $\left(K_{M}\right.$ and $\left.V_{\max }\right)$ associated with this activity and compared them to those of horseradish peroxidase. The $K_{M}$ of the new material was lower than that of the reference enzyme, demonstrating the greater affinity of this material to the studied substrates. This observation highlights the fact that the incorporation of catalysts, even chemical ones, into such flexible materials allows one to achieve catalytic behaviours close to those of enzymes with increased specificity and selectivity. It should also be added that while the incorporation of metallic nanoparticles has not led to major changes in MOF morphology, that of the enzyme has led to crystallisation in a different form, which shows how materials of this class can adapt to the incorporation of various molecules. However, catalyst incorporation did not change the crystalline phase, possibly because the pore size of the employed MOF (ZIF-8; $11.6 \AA$ ) did not allow the direct incorporation of enzymes and nanoparticles. On the other hand, the coating of enzymes with ZIF-8 has been shown to protect them against biological, thermal, and chemical degradation while maintaining their bioactivity. ${ }^{42}$ For this reason, the authors chose to synthesise their MCHM by coprecipitation of catalysts with MOFs. However, this approach limits the number of catalysts and materials that can be used, 
as the former must resist the conditions used to synthesise the latter. The above technique also makes it difficult to precisely organise the distribution of catalysts within the material, and the use of MOFs with larger pore sizes may therefore be more interesting, as we will see below. The first interesting property of this new MCHM is the more efficient use of glucose oxidase (which is strongly inhibited by $\mathrm{H}_{2} \mathrm{O}_{2}$ ) due to the suppression of its denaturation during the reaction through reduction with NiPd nanoparticles. The authors also observed that in the presence of nanoparticles, enzymatic activity exceeded that observed when the enzyme was immobilised in the MOF on its own. Therefore, the authors hypothesised that this "higher catalytic activity can be attributed to the hollow nanoparticle structure of NiPd in the material, which may help capture more glucose oxidase molecules in ZIF-8" without verifying the amount of enzyme immobilised in either case. It is therefore rather likely that this increase in catalytic activity is indeed due to the preservation of the enzyme and its rapid uptake by the nanoparticles to result in the described synergistic effect. Although the nanoparticle presence was not originally intended for an additional catalytic step, the authors succeeded in achieving a catalytic cascade using $o$-phenylenediamine as a substrate for the peroxidase activity of the material. This system demonstrated the potential of the above MCHM for the colorimetric detection of glucose in solution, a useful analytical application for medical and food processing environments. The authors also used this concept to study the electrocatalytic activity of their new MCHM for oxygen reduction, a property of great interest for the fabrication of electrochemical biosensors for glucose detection.

Since this first example, several others have been synthesised with MOFs as supports to improve the synergy between catalysts. However, in most cases, the chemical catalysts (metallic nanoparticles) were incorporated into the material, whereas the enzymes were immobilised on the surface. ${ }^{36,43}$ Again, this behaviour was ascribed to the use of a MOF (UiO-66) with relatively small pores (largest dimension $=21 \AA$ ). However, a study published in early 2020 provides an example of a MOFbased MCHM featuring enzymes incorporated within the material pores. ${ }^{44}$ This breakthrough was achieved through the use of polyvinylpyrrolidone (PVP), a non-ionic amphiphilic polymer, for the generation of mesopores (>20 nm) during the crystallisation of a MOF based on the structure of cobaltcontaining zeolite imidazolates (ZIF-67). PVP was used as a bulky polymer that participated in metal coordination during the crystallisation of ZIF-67 and thus partially disrupted the catenation of the imidazole-Co(II) complex to afford a crystal structure with abundant mesopores, more commonly referred to as holes. Pd nanocrystals already complexed with PVP during MOF synthesis were added and easily introduced into the material structure, as they were logically located at the level of the formed mesopores (Fig. 7). The same approach was also tested using Pd nanocrystals, again leading to the successful incorporation of the metal centres into the MOF. Even more interestingly, at the end of the synthesis, the authors noticed the presence of $\mathrm{Co}$ (II) cations showing a coordination defect in imidazole groups caused by the use of PVP. What is remarkable here is that these metal centres also exhibited catalytic activity, which, however, was different from that of Pd nanoparticles. Finally, as far as the enzyme is concerned, the sufficiently large size of the mesopores of the thus formed material allowed the incorporation of Candida antarctica lipase A (CalA, dimensions $=4.2 \mathrm{~nm} \times 5.6 \mathrm{~nm} \times 6.3 \mathrm{~nm}$ ).
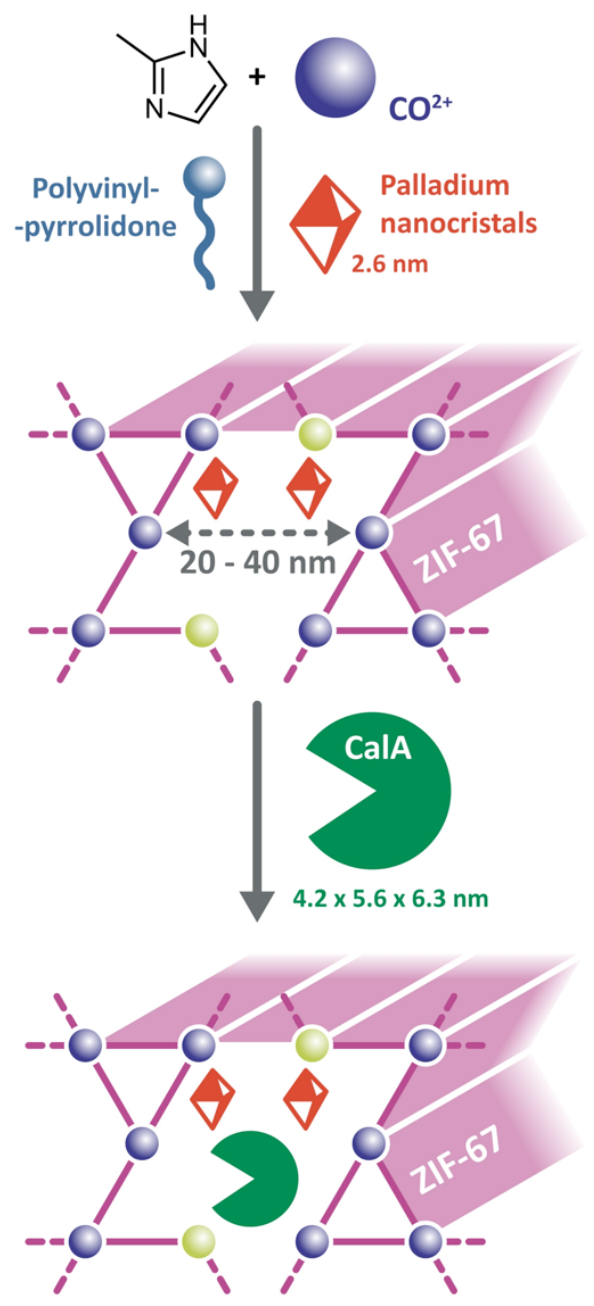

Fig. 7. Synthesis of a MOF-67-based MCHM with mesopores allowing the immobilisation of relatively large catalysts within its structure. The first step corresponds to crystal growth in the presence of $\mathrm{Co}^{2+}$ ions, 2-methylimidazole, and PVP $\left(M_{\mathrm{W}}=10000 \mathrm{Da}\right)$. The coordination-deficient catalytically active $\mathrm{Co}^{2+}$ cations formed in the presence of PVP are shown in light green. Pd nanocrystals were introduced during growth and immobilised in the formed mesopores. In the second step, an enzyme (CalA) was introduced into the mesopores by simple incubation of the material in an aqueous solution at room temperature (adapted from Dutta et al., ${ }^{44}$ translated from L'Actualité Chimique, $\mathrm{N}^{\circ} 454$, September 2020, with agreement).

This method maintained the activity of the enzyme, and the authors even observed a protective effect of the material against denaturation, as expected. Thus, the authors succeeded in creating the first MCHM with three distinct catalytic activities. The synthetic potential of this MCHM was illustrated by applying it to the formation of nitroalcohols (Henry's reaction) catalysed by unsaturated $\mathrm{Co}$ (II) sites followed by the Pd nanocrystal-catalysed racemisation of the unwanted enantiomer, while the other enantiomer was then acylated by lipase (Fig. 8). 


\section{TUTORIAL REVIEW}

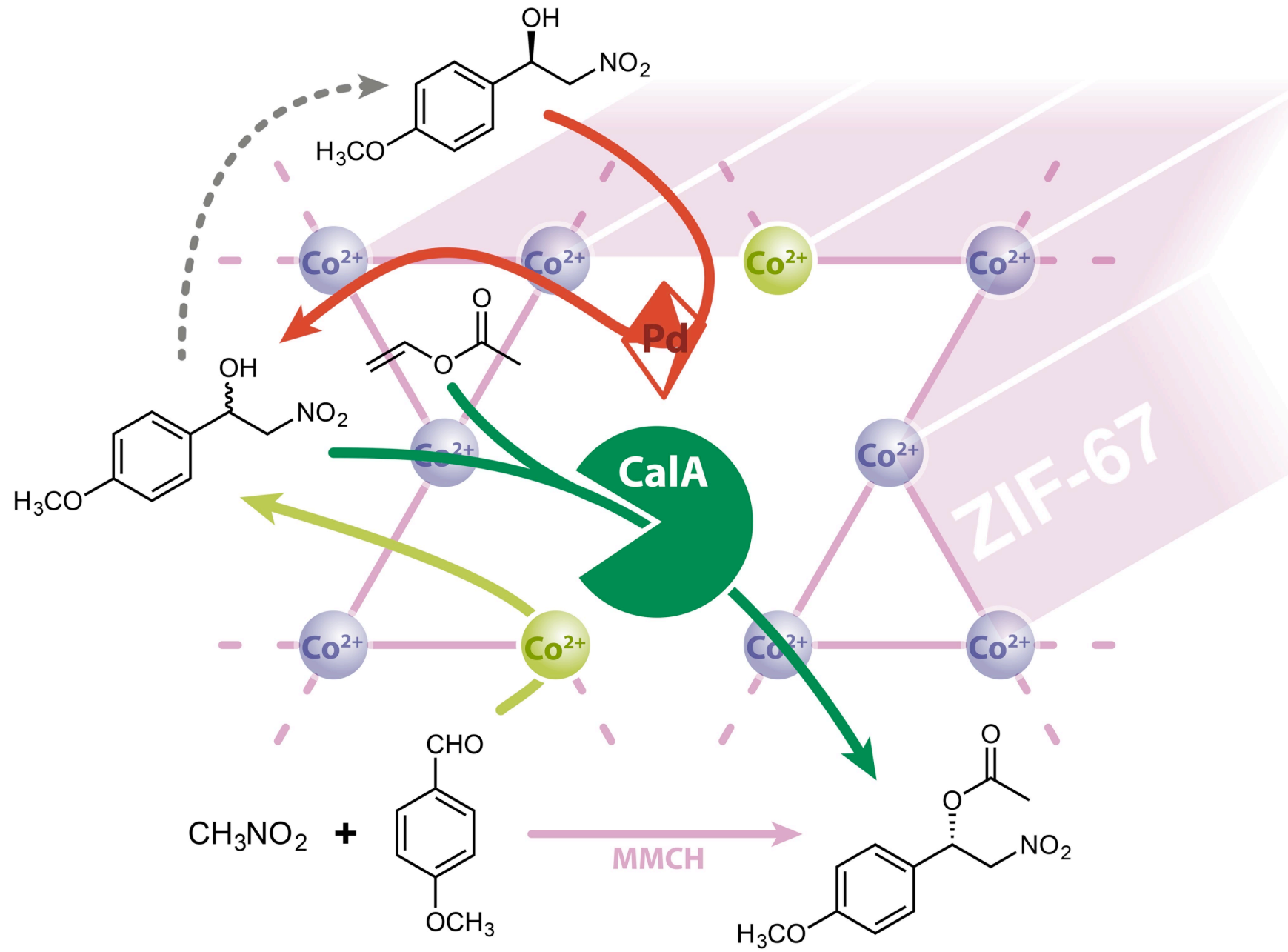

Fig. 8. Synthesis of (S)-1-(4-methoxyphenyl)-2-nitroethyl acetate from 4-methoxybenzaldehyde, nitromethane, and vinyl acetate using an MCHM based on ZIF-67 (Co2+), CalA, and Pd nanocrystals. The reaction was carried out at room temperature in a 4:1 (v/v) tetrahydrofuran:toluene mixture (figure adapted from Dutta et al., ${ }^{44}$ translated from L'Actualité Chimique, $\mathrm{N}^{\circ} 454$, September 2020, with agreement).

In addition, the authors applied their synthetic strategy to a variety of aldehydes, obtaining conversions and ee values of $>99 \%$ in all cases. In addition, the catalyst showed good recyclability with a moderately affected residual activity ( $>89 \%$ ), with a yield of $86 \%$ and an $e e>80 \%$ obtained after five successive recycling steps. It should be noted, however, that these efficiencies were obtained after a slight increase in reaction time from 20 to $35 \mathrm{~h}$ between the first and fifth cycles. Finally, the authors were interested in the synergistic effect between the catalysts co-immobilised in the MOF. By studying the different combinations of free and immobilised catalysts, they showed that only the MCHM exhibited such a high performance (efficiency and stereoselectivity). The second best combination, featuring enzymes and $\mathrm{Pd}$ nanoparticles immobilised in separate MOF crystals, achieved a yield of 60\% with an ee of $35 \%$. All other combinations led to less satisfactory results. This finding confirms the usefulness of coimmobilising catalysts in MCHMs to generate synergistic effects between these catalysts. Finally, the authors also showed that although the presence of enzymes in the mesopores could lead to their partial blockage, the micropores of the crystalline structure of ZIF-67 allowed the substrates and products to freely circulate within the material. This result demonstrates the benefits offered by this tunable-pore-size structure for the construction of hybrid materials in which the immobilised catalytic sites have radically different sizes and an appropriate (or even optimised) environment. In addition, several other MOFs with large pores (e.g., PCN-333, PCN-888, and the NU- 
100x family) have been developed and successfully used for enzyme immobilisation, which promises a bright future for this type of materials in the development of MCHMs. ${ }^{42}$ Finally, MOFs are obviously not the only promising family of flexible nanomaterials for the co-immobilisation of enzymes and metal catalysts, the other family being their organic equivalents, namely COFs, even if their more rigid structures limit their adaptability for MCHM creation. One can also mention other classes of even less common materials such as metalbiosurfactant nanocomposites, which have been used for the synthesis of an MCHM through the immobilisation of a lipase and a Shvo complex, a Ru catalyst, for dynamic kinetic resolution reactions. ${ }^{45}$

\section{Call for interdisciplinary collaboration}

In conclusion, we hope to have demonstrated that the development of multi-catalytic hybrid materials offers many advantages for catalytic synthesis. The great versatility of structures and catalysts that can theoretically be developed increases the diversity of accessible compounds tenfold while offering substantial atom and energy savings, particularly in regard to the biorefineries of tomorrow. However, this field of research is still very young, and many challenges remain to be addressed in terms of chemical and biological catalysts per se and in the field of materials and the development of innovative processes to achieve the effective combination of the latter. In particular, while the examples presented here involve only the combination of biological and chemical catalysts, the interest in synthetizing new MCHMs is not limited to these two fields, but also concerns all fields of sciences applied to catalysis. Hopefully, other types of MCHMs will soon be developed, involving other species, starting with chemical catalysts beyond metallic nanoparticles. Indeed, many studies have demonstrated the very close relationship that can exist between catalysts and the materials used as supports, leading to the production of very innovative multi-catalytic chemical materials for exemple. ${ }^{46,47}$ As these often exhibit new catalytic properties of their own, linked to the presence of additional steric and especially electronic effects, we can assume that they will pave the way for a whole new generation of MCHMs with abilities and reaction conditions radically different from those presented here, thus multiplying even more the scope of possibilities of this young discipline. ${ }^{3}$ Priority should therefore be given to promoting communication and collaboration between chemists and biologists, whose current concerns are still quite far apart (with some reluctance on the part of biologists towards chemistry and vice versa). Indeed, there is now a consensus that future innovations will benefit from an intensification of interdisciplinary research efforts. Thus, there is no doubt that the strengthening of collaborations between these two worlds will effectively open up a new field of knowledge and lead to the implementation of new industrial processes to meet the societal challenges of the coming decades.

\section{Conflicts of interest}

There are no conflicts to declare.

\section{Acknowledgements}

The authors thank the REALCAT platform funded by the French government grant managed by the French National Research Agency (ANR) as part of the "Investments for the Future" program (ANR-11-EQPX-0037). We also acknowledge the Hauts-de-France region, the ERDF, the Ecole Centrale de Lille, and the Fondation Centrale Initiatives for financial support with the acquisition of the REALCAT platform equipment. This study was also supported by the French government through the Programme d'Investissements d'Avenir (I-SITE ULNE/ANR-16IDEX-0004 ULNE) managed by the National Research Agency. Chevreul Institute (FR 2638), Ministère de l'Enseignement Supérieur, de la Recherche et de l'Innovation, Région Hauts-deFrance and FEDER are acknowledged for supporting and funding partially this work.

\section{Notes and references}

1 P. Anastas and J. Warner, Green Chemistry: Theory and Practice, Oxford University Press, Oxford, New York, 2000.

2 A. Weller and S. Fletcher, Catalysis - Department of Chemistry, https://www.chem.ox.ac.uk/catalysis.aspx, (accessed September 27, 2020).

3 E. Heuson and F. Dumeignil, Catal. Sci. Technol., 2020, 10, 7082-7100.

4 X. Li, X. Cao, J. Xiong and J. Ge, Small, 2019, 1902751.

5 R. Kourist and J. González-Sabín, ChemCatChem, 2020, 12, 1903-1912.

6 R. Ye, J. Zhao, B. B. Wickemeyer, F. D. Toste and G. A. Somorjai, Nat. Catal., 2018, 1, 318-325.

7 Y. Wang, H. Ren and H. Zhao, Crit. Rev. Biochem. Mol. Biol., 2018, 53, 115-129.

8 S. Schmidt, K. Castiglione and R. Kourist, Chem. - Eur. J., 2018, 24, 1755-1768.

9 F. Rudroff, M. D. Mihovilovic, H. Gröger, R. Snajdrova, H. Iding and U. T. Bornscheuer, Nat. Catal., 2018, 1, 12-22.

10 N. Ríos-Lombardía, J. García-Álvarez and J. González-Sabín, Catalysts, 2018, 8, 75.

11 F. Dumeignil, M. Guehl, A. Gimbernat, M. Capron, N. L. Ferreira, R. Froidevaux, J.-S. Girardon, R. Wojcieszak, P. Dhulster and D. Delcroix, Catal. Sci. Technol., 2018, 8, 57085734.

12 J. Wu, S. Li and H. Wei, Chem. Commun., 2018, 54, 6520-6530.

13 S. Zhang, C. Wang, H. Chang, Q. Zhang and Y. Cheng, Sci. Adv., 2019, 5, eaaw4252.

14 C. Wang, Q. Zhang, X. Wang, H. Chang, S. Zhang, Y. Tang, J. Xu, R. Qi and Y. Cheng, Angew. Chem., Int. Ed., 2017, 56, 67676772.

15 J. M. Foulkes, K. J. Malone, V. S. Coker, N. J. Turner and J. R. Lloyd, ACS Catal., 2011, 1, 1589-1594.

16 T. Douglas, D. P. E. Dickson, S. Betteridge, J. Charnock, C. D. Garner and S. Mann, Science, 1995, 269, 54-57.

17 F. C. Meldrum, V. J. Wade, D. L. Nimmo, B. R. Heywood and S. Mann, Nature, 1991, 349, 684-687.

18 B. H. San, S. Kim, S. H. Moh, H. Lee, D.-Y. Jung and K. K. Kim, Angew. Chem., Int. Ed., 2011, 50, 11924-11929.

19 X. Li, Y. Cao, K. Luo, Y. Sun, J. Xiong, L. Wang, Z. Liu, J. Li, J. Ma, J. Ge, H. Xiao and R. N. Zare, Nat. Catal., 2019, 2, 718-725. 
20 M. Filice, M. Marciello, M. del Puerto Morales and J. M. Palomo, Chem. Commun., 2013, 49, 6876-6878.

21 K. P. J. Gustafson, T. Gorbe, G. de Gonzalo, N. Yuan, C. L. Schreiber, A. Shchukarev, C.-W. Tai, I. Persson, X. Zou and J.E. Backvall, Chem.-Eur. J., 2019, 25, 9174-9179.

22 T. Görbe, K. P. J. Gustafson, O. Verho, G. Kervefors, H. Zheng, X. Zou, E. V. Johnston and J.-E. Bäckvall, ACS Catal., 2017, 7, 1601-1605.

23 Z. Li, Y. Ding, X. Wu, J. Ge, P. Ouyang and Z. Liu, RSC Adv., 2016, 6, 20772-20776.

24 D. Jagadeesan, D. Vernekar, S. Gupta and G. Jaiswal, Proc. Indian Natl. Sci. Acad., 2018, 85, 23-41.

25 R. J. Davis, J. Catal., 2003, 216, 396-405.

26 A. Corma and A. Martinez, Adv. Mater., 1995, 7, 137-144.

27 P. N. R. Vennestrøm, E. Taarning, C. H. Christensen, S. Pedersen, J.-D. Grunwaldt and J. M. Woodley, ChemCatChem, 2010, 2, 943-945.

28 V. Smeets, W. Baaziz, O. Ersen, E. M. Gaigneaux, C. Boissière, C. Sanchez and D. P. Debecker, Chem. Sci., 2020, 11, 954-961.

29 S. P. de Souza, R. A. C. Leão, J. F. Bassut, I. C. R. Leal, S. Wang, Q. Ding, Y. Li, F. L.-Y. Lam, R. O. M. A. de Souza and I. Itabaiana Jr, Tetrahedron Lett., 2017, 58, 4849-4854.

30 K. Engström, E. V. Johnston, O. Verho, K. P. J. Gustafson, M. Shakeri, C.-W. Tai and J.-E. Bäckvall, Angew. Chem., Int. Ed., 2013, 52, 14006-14010.

31 N. Zhang, R. Huebner, Y. Wang, E. Zhang, Y. Zhou, S. Dong and C. Wu, ACS Appl. Nano Mater., 2018, 1, 6378-6386.

32 A. K. Ganai, P. Shinde, B. B. Dhar, S. S. Gupta and B. L. V. Prasad, RSC Adv., 2013, 3, 2186-2191.

33 R. Jin, D. Kong, X. Zhao, H. Li, X. Yan, F. Liu, P. Sun, D. Du, Y. Lin and G. Lu, Biosens. Bioelectron., 2019, 141, UNSP 111473.

34 T. Xue, B. Peng, M. Xue, X. Zhong, C.-Y. Chiu, S. Yang, Y. Qu, L. Ruan, S. Jiang, S. Dubin, R. B. Kaner, J. I. Zink, M. E. Meyerhoff, X. Duan and Y. Huang, Nat. Commun., 2014, 5, 3200.

35 Y. Wang, N. Zhang, R. Hübner, D. Tan, M. Löffler, S. Facsko, E. Zhang, Y. Ge, Z. Qi and C. Wu, Adv. Mater. Interfaces, 2019, 6, 1801664.

36 Y. Wang, N. Zhang, E. Zhang, Y. Han, Z. Qi, M. B. AnsorgeSchumacher, Y. Ge and C. Wu, Chem. - Eur. J., 2019, 25, 17161721.

37 S. Abednatanzi, P. Gohari Derakhshandeh, H. Depauw, F.-X. Coudert, H. Vrielinck, P. Van Der Voort and K. Leus, Chem. Soc. Rev., 2019, 48, 2535-2565.

38 A. Karmakar and A. J. L. Pombeiro, Coord. Chem. Rev., 2019, 395, 86-129.

39 A. Dhakshinamoorthy, A. M. Asiri and H. Garcia, Catal. Sci. Technol., 2016, 6, 5238-5261.

40 L. Chen and Q. Xu, Matter, 2019, 1, 57-89.

41 Q. Wang, X. Zhang, L. Huang, Z. Zhang and S. Dong, Angew. Chem., Int. Ed., 2017, 56, 16082-16085.

42 X. Lian, Y. Fang, E. Joseph, Q. Wang, J. Li, S. Banerjee, C. Lollar, X. Wang and H.-C. Zhou, Chem. Soc. Rev., 2017, 46, 33863401.

43 Y. Wu, J. Shi, S. Mei, H. A. Katimba, Y. Sun, X. Wang, K. Liang and Z. Jiang, ACS Catal., 2020, 9664-9673.

44 S. Dutta, N. Kumari, S. Dubbu, S. W. Jang, A. Kumar, H. Ohtsu, J. Kim, S. H. Cho, M. Kawano and I. S. Lee, Angew. Chem., Int. Ed., 2020, 59, 3416-3422.

45 H. Li, C. Qiu, X. Cao, Y. Lu, G. Li, X. He, Q. Lu, K. Chen, P. Ouyang and W. Tan, ACS Appl. Mater. Interfaces, 2019, 11, 1571815726.

46 D. Jagadeesan, Appl. Catal., A, 2016, 511, 59-77.

47 M. J. Climent, A. Corma, S. Iborra and M. J. Sabater, ACS Catal., 2014, 4, 870-891. 